\title{
Mesenchymal stem cells modified with miR-126 release angiogenic factors and activate Notch ligand Delta-like-4, enhancing ischemic angiogenesis and cell survival
}

\author{
FENG HUANG $^{1 *}$, XIAO ZHU ${ }^{2 *}$, XIN-QUN HU ${ }^{1}$, ZHEN-FEI FANG $^{1}$, LIANG TANG ${ }^{1}$, \\ XIAO-LING LU ${ }^{1}$ and SHENG-HUA ZHOU ${ }^{1}$ \\ ${ }^{1}$ Department of Cardiology, ${ }^{2}$ Institute of Metabolism and Endocrinology, The Second Xiangya Hospital of \\ Central South University, Changsha, Hunan 410011, P.R. China
}

Received September 18, 2012; Accepted November 9, 2012

DOI: $10.3892 / \mathrm{ijmm} .2012 .1200$

\begin{abstract}
The endothelial cell-specific microRNA (miRNA), miR-126, is considered a master regulator of physiological angiogenesis. Transplanted mesenchymal stem cells (MSCs) release soluble factors contributing to neoangiogenesis and cardiac repair. Therefore, we hypothesized that the overexpression of miR-126 may prolong MSC survival and enhance the cell secretome, thereby improving post-infarction angiogenesis and cardiac function. In this study, MSCs harvested from male C57BL/6 mouse bone marrow were infected in vitro with miR-126 (MSC ${ }^{\text {miR-126}}$ ) by using recombinant lentiviral vectors; the control cells were either non-transfected or transduced with mock vectors (MSC ${ }^{\text {null }}$ ). The results showed an increased secretion of angiogenic factors and a higher resistance against hypoxia in MSC ${ }^{\text {miR-126 }}$ compared with the control cells. The expression of the Notch ligand Delta-like (Dll)-4 in the MSC ${ }^{\text {miR-126 }}$ group was
\end{abstract}

Correspondence to: Professor Sheng-Hua Zhou, Department of Cardiology, The Second Xiangya Hospital of Central South University, No. 139 Renmin Road, Changsha, Hunan 410011, P.R. China

E-mail: zhougqin@yeah.net

${ }^{*}$ Contributed equally

Abbreviations: MSCs, mesenchymal stem cells; MI, myocardial infarction; VEGF, vascular endothelial growth factor; bFGF, basic fibroblast growth factor; miRNAs or miRs, microRNAs; 3'-UTR, 3'-untranslated regions; SPRED1, Sprouty-related protein 1; PIK3R2, phosphoinositol-3 kinase regulatory subunit 2; Dll, Delta-like; Jag, jagged; NICD, Notch intracellular domain; bHLH, basic helix-loophelix; PBS, phosphate-buffered saline; shRNA, short hairpin RNA; FITC, fluorescein isothiocyanate; eGFP, enhanced green fluorescent protein; LV, left ventricular; LAD, left anterior descending artery; LVID, left ventricular internal dimensions; EF, ejection fraction; FS, fractional shortening; EDV, end-diastolic LVID; ESV, end-systolic LVID; ANOVA, analysis of variance; MTT, (3,4,5-dimethylthiazol-2yl)-2,5-diphenyltetrazolium bromide; $\mathrm{Ct}$, threshold cycles; EGF, epidermal growth factor; MAPK, mitogen-activated protein kinase

Key words: mesenchymal stem cells, miR-126, Delta-like-4, ischemic angiogenesis, tubulogenesis, angiogenic factor also increased. For in vivo experiments, $\mathrm{MSC}^{\mathrm{miR}-126}$ cultures were intramyocardially injected into the infarct region of the hearts of female C57BL/6 mice (an acute myocardial infarction model) who had undergone ligation of the left anterior descending coronary artery. The survival of MSC ${ }^{\text {miR-126 }}$ cultures, determined by Sry expression, was increased at 7 days after transplantation.

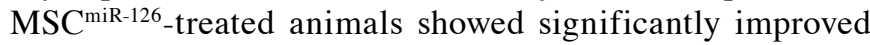
cardiac function as assessed by echocardiography 2 weeks later. The expression levels of angiogenic factors and Dll-4 in the infarcted myocardium were further increased by $\mathrm{MSC}^{\mathrm{miR}-}$ 126 compared with MSCs or MSC ${ }^{\text {null }}$ cultures. Furthermore, fluorescent microsphere and histological studies revealed that myocardial blood flow and microvessel density were signifi-

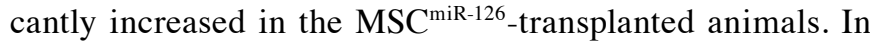
addition, we found increased immature vessel proliferation following the transplantation of MSC ${ }^{\text {miR-126 }}$ cultures in which the expression of Dll-4 had been knocked down. However, blood vessels with lumen were barely detected, which indicated that Dll-4 plays a key role in tubulogenesis. We conclude that the transplantation of MSCs overexpressing miR-126 can further enhance functional angiogenesis in the ischemic myocardium possibly by the secretion of angiogenic factors and the activation of Dll-4, thus increasing MSC survival. Therefore, MSCs modified with miR-126 may represent a novel and efficient therapeutic approach for ischemic angiogenesis and the improvement of cardiac function.

\section{Introduction}

Ischemic heart disease is the most common cause of mortality worldwide despite continued improvements in the prevention and treatment of coronary artery disease. However, the majority of patients are not qualified for the conventional revascularization techniques of balloon angioplasty and stenting, or coronary artery bypass grafting. Over the last decade, stem cell transplantation has been considered as a promising approach for the recovery of heart function in ischemic disease. Mesenchymal stem cells (MSCs) can be easily isolated and expanded, and have a stable genetic background and a low risk of immunorejection. Furthermore, stem cells possess the capacity of multilineage differentiation and bioenergetic modulation $(1,2)$. As a result, 
MSCs are used as seeding cells in tissue engineering and stem cell therapy. They have been used in both experimental and clinical settings to attenuate infarct size expansion, enhance myocardial regeneration and cardiac function (1-5). Previous studies have indicated that the functional benefits observed after MSC transplantation in animal models of cardiac injury may be related to the secretion of soluble factors that, acting in a paracrine fashion, attenuate pathological ventricular remodeling, induce angiogenesis and promote myocardium regeneration $(1,2,4,6)$. Angiogenesis is essential for cardiac repair following myocardial infarction (MI), when collateral vessels form at the site of the infarct and maintain blood flow to ischemic tissue (7). MSCs may differentiate into vascular endothelial-like cells and incorporate into growing new blood vessels by the paracrine release of a series of angiogenic cytokines, including vascular endothelial growth factor (VEGF) and basic fibroblast growth factor (bFGF) (8). However, the low survival rate and angiogenic potential of transplanted cells in the ischemic myocardium influence the outcome of MSC transplantation for the treatment of ischemic heart disease.

microRNAs (miRNAs or miRs) are naturally occurring RNAs that are transcribed in the nucleus, processed by the RNases Drosha/DGCR8 and Dicer into mature 22-nucleotide RNAs that bind to complementary target mRNAs through Watson-Crick base pairing between the miRNA 'seed region' and sequences commonly located in the 3'-untranslated regions (3'-UTR) (9-12). As they can regulate numerous genes, miRNAs are considered as master regulators of cellular processes, such as cellular differentiation and organogenesis. The endothelial cellspecific miRNA, miR-126, has been shown to positively regulate the response of endothelial cells to VEGF and to improve angiogenesis partly by directly repressing negative regulators of the VEGF pathway, including the Sprouty-related protein 1 (SPRED1), and phosphoinositol-3 kinase regulatory subunit 2 (PIK3R2) (13). Thus, the overexpression of miR-126 relieves the repressive influence of SPRED1/PIK3R2 on the signaling pathways activated by VEGF and bFGF, favoring angiogenesis. It plays an essential role in neoangiogenesis following MI and in the maintenance of vascular integrity. The targeted deletion of miR-126 causes a loss of vascular integrity in mice and zebrafish during development and results in defective angiogenesis (14). A recent study showed that the blood flow-induced upregulation of miR-126 by the mechanosensitive transcription factor, Klf2, in endothelial cells activated the VEGF signaling pathway and led to sprouting and remodeling of the aortic arch in developing zebrafish (15). These findings establish the involvement of miR-126 in potently promoting physiological angiogenesis and enhancing blood flow to ischemic tissue.

In addition to VEGF, many Notch pathway components are expressed and play a critical role during vessel development (16-19). In mammals, the Notch pathway involves 5 transmembrane ligands, Delta-like (D1l)-1, -3, -4, Jagged (Jag)-1 and Jag-2, and 4 transmembrane receptors, Notch-1, $-2,-3$ and -4 . Notch receptor activation results in the cleavage of the Notch intracellular domain (NICD) and translocation to the nucleus, thereby activating downstream target genes, such as basic helix-loop-helix (bHLH) protein $(18,20)$. Functional studies have provided us with convincing evidence that the Notch pathway is involved in a variety of cell fate decisions and regulates many biological processes, particularly angiogenesis.
Therapies combining genes and stem cells hold promise for the treatment of ischemic heart disease. In particular, MSCs are excellent carriers of therapeutic genes to the ischemic zone. Accordingly, we hypothesized that the transplantation of MSCs modified with miR-126 may be superior to MSCs, increasing the stem cell paracrine activity and inducing the Notch pathway to promote ischemic angiogenesis, thereby enhancing cardiac functional recovery.

\section{Materials and methods}

Animal ethics. All procedures were performed in compliance with the guidelines for the Care and Use of Laboratory Animals published by the National Institutes of Health (NIH publication no. 85-23, revised 1996) and approved by the Animal Care and Use Committee of the Second Xiangya Hospital, Central South University. The investigators responsible for molecular, histological and functional studies were blinded to the treatment groups.

Isolation and primary cultivation of MSCs. Six-week-old male C57BL/6 mice were sacrificed by cervical dislocation. The hind legs and vertebrae were dissected and carefully separated from the adherent tissues. After the tips of each bone were removed, bone marrow was collected by flushing out the content of the femurs and tibia with phosphate-buffered saline (PBS). The collected cells were plated at $1 \times 10^{6}$ cells $/ \mathrm{ml}$ in $100-\mathrm{mm}$ plastic dishes and cultured in complete medium, consisting of Dulbecco's modified Eagle's medium/Nutrient Mixture F12 (DMEM/F12), $10 \%$ fetal bovine serum and $1 \%$ penicillin/streptomycin (all from Gibco). The passaged cells were analyzed by fluorescence-activated cell sorting as previously described (21). After blocking for non-specific binding with buffer containing $1 \%$ bovine serum albumin, the cells were incubated for $20 \mathrm{~min}$ at $4^{\circ} \mathrm{C}$ with the following antibodies (Santa Cruz Biotechnology, Inc.): anti-CD29-fluorescein isothiocyanate (FITC), anti-CD34-FITC, anti-c-kit-FITC, anti-CD45-FITC, anti-CD90-FITC and anti-CD105-FITC. The differentiation of MSCs in vitro towards the adipogenic and osteogenic lineage was carried out as previously described $(22,23)$.

Recombinant lentiviral vector construction, cell infection and stable cell line generation. To construct recombinant lentiviral miRNA expression vectors, mature miR-126, TRE promoter and enhanced green fluorescent protein (eGFP) sequences were inserted into plasmids for the generation of pUp-TRE, pDown-miR126 and pTail-IRES/eGFP. pLV.EX3d.P/ puro-TRE $>$ miR126 $>$ IRES/eGFP was obtained with the incubation of donors and accepter vectors catalyzed by LR clonase (Gateway ${ }^{\circledR}$ LR Clonase ${ }^{\text {TM }}$ Plus Enzyme Mix; Invitrogen). The envelope helper plasmids, pLV/helper-SL3, pLV/helper-SL4, pLV/helper-SL5, with pLV. EX3d.P/puro-TRE-miR126-IRES/ eGFP or pLVrtTA/neo, were co-transfected into 293T cells with Lipofectamine 2000 according to the manufacturer's instructions (Invitrogen), to produce Lenti-miR126-eGFP/puro or Lenti-rtTA/neo, respectively. In order to perform lentiviral infections, MSCs were first treated by Lenti-rtTA/neo; $48 \mathrm{~h}$ later, infected cells were selected in $0.5 \mathrm{mg} / \mathrm{ml}$ neomycin and fresh medium every 2 days. Selection was terminated when the control cells were completely dead and antibiotic-free medium was used for propagation. Neomycin-resistant cells 
were infected by Lenti-miR126-eGFP/puro and grown with $2 \mu \mathrm{g} / \mathrm{ml}$ puromycin. Double-resistant cells were then ultimately obtained, and $2 \mu \mathrm{g} / \mathrm{ml}$ doxycycline was added to medium. The overexpression of miR-126 was achieved. MSCs infected with miR-126 recombinant lentiviral vectors encoding eGFP were termed MSC $^{\text {miR-126 }}$, and MSCs infected with mock vectors carrying eGFP but without miR-126 were termed MSC $^{\text {null }}$, which was used to determine whether the mock vectors influence the cells in a paracrine manner and the signaling molecules involved compared to the control MSCs.

To determine whether Dll-4 plays a role in tubulogenesis, MSC $^{\text {miR-126 }}$ colony was infected with lentiviral constructs encoding short hairpin RNA (shRNA) to knockdown Dll-4. Three types of plasmids, pAJ-U6-shRNA-CMV-Puro/eGFP, psPAX2 (gag/pol element), and pMD2.G (VSVG element), were transfected into $293 \mathrm{~T}$ cells according to the instructions for Lipofectamine 2000 (Invitrogen). After $48 \mathrm{~h}$ of transduction, the stable shRNA line was selected.

Quantitative real-time PCR to identify the efficiency of miR-126 transfection. Total RNA was extracted from each sample with TRIzol reagent (Invitrogen) according to the manufacturer's instructions. Complimentary DNA was synthesized in a $20-\mu 1$ reaction mixture using the SuperScript III First-Strand Synthesis System for RT-PCR (Invitrogen). The expression of miR-126 in the transduced cells was detected by real-time PCR using the All-in-One ${ }^{\mathrm{TM}}$ miRNA quantitative real-time PCR Detection kit and All-in-One ${ }^{\mathrm{TM}}$ miRNA qPCR Primer (from GeneCopoeia). The primer sequence was 5'-CATT ATTACTTTTGGTACGCGAAA-3'. miR-126 transcript levels were normalized to the control, U6 mRNA, whose primer sequence was 5'-TCGTGAAGCGTTCCATATTTTTAA-3'.

VEGF and bFGF secretion from miR-126-modified MSCs under hypoxic conditions. After the MSCs, mock-vectorinfected MSCs and miR-126-transduced MSCs completed adherence, they were incubated for $24 \mathrm{~h}$ at $37^{\circ} \mathrm{C}$ in a humidified modular hypoxia chamber (Billups Rothenberg, Inc.) containing 95\% nitrogen and 5\% carbon dioxide $(\mathrm{n}=4$ in each group). Subsequently, the conditioned medium was collected for analysis. Commercial VEGF or bFGF enzyme-linked immunosorbent assay (ELISA) kits (R\&D Systems Inc., Minneapolis, MN, USA) were used to quantify the concentration of VEGF and bFGF in each sample. The supernatant from MSCs cultured under normal conditions was used as the control. Each experiment was repeated 3 times.

Creation of MI model and cell transplantation. Eight-week-old female C57BL/6 mice weighing $20 \pm 2 \mathrm{~g}$ underwent ligation of the left coronary artery to produce MI. After being anesthetized by an intraperitoneal injection of $150 \mu \mathrm{l}(1 \%)$ pentobarbital sodium (Merck, Darmstadt, Germany), the mice were orally intubated with a 1.0-mm OD intubation cannula and connected to a small animal volume-control ventilator (HES-HA MiniVent 845; Harvard Apparatus, Holliston, MA). The left anterior descending artery (LAD) was ligated 2-3 $\mathrm{mm}$ from its origin between the pulmonary artery conus and the left atrium by using 8-0 sutures. Mice were subjected to electrocardiographs using the RM6240BD system (Chengdu Instrument Company, Changdu, China) to determine MI. After pumping out the gas in the chest with a 1-ml syringe to create negative pressure, the intercostal space incision was closed with 6-0 sutures, and skin incision was closed with 5-0 sutures.

One week after coronary ligation, the surviving mice were randomly divided into 4 groups (PBS group, MSC group, $\mathrm{MSC}^{\text {null }}$ group and $\mathrm{MSC}^{\text {miR-126 }}$ group; 20 in each), and received re-thoracotomy and an intramyocardial injection of PBS, MSC, MSC $^{\text {null }}$ or MSC ${ }^{\text {miR-126 }}$. Each received an injection of $5 \times 10^{6}$ cells $/ 100 \mu 1$ in PBS or PBS alone with a total volume of $50 \mu \mathrm{l}$ at 5 sites (basal anterior, mid-anterior, mid-lateral, apical anterior and apical lateral) in the peri-infarcted area, with $10 \mu \mathrm{l}$ at each site. The chest was then closed and the animals were allowed to recover.

Assessment of cardiac function by echocardiography. Transthoracic echocardiographic studies were performed by an experienced blinded cardiologist 14 days after the cell transplantation using an echocardiographic system (Sonos 5500; Hewlett-Packard, Andover, MA) equipped with a $15.0 \mathrm{MHz}$ transducer. For analysis of left ventricular (LV) function, left ventricular internal dimensions (LVID) were measured. The echo transducer was placed on the left hemithorax and views were recorded. Two-dimensional images were obtained at the midpapillary muscle level. LV ejection fraction (EF; LVEF) $(\%)=($ EDV - ESV $) /$ EDV x $100 \%$, fractional shortening $(\mathrm{FS})(\%)=[($ LVIDD - LVIDS $) /$ LVIDD $] \times 100 \%$, where D stands for diastole, $S$ for systole and EDV and ESV stand for end-diastolic and end-systolic LVID, respectively. The images were obtained in each view and each parameter was measured from 3 consecutive beat cycles in each image.

Western blot analysis. Cells of each group were harvested after being cultured for $72 \mathrm{~h}$. Cells of MSC, MSC ${ }^{\text {null }}$ and $\mathrm{MSC}^{\text {miR-126 }}$ colonies were extracted to examine the alteration of Notch signaling compare to MSCs. At 1 and 2 weeks after cell transplantation, the infarcted areas in the PBS, MSC, MSC ${ }^{\text {null }}$ and $\mathrm{MSC}^{\mathrm{miR} 126}$ groups (10 in each group) were excised for VEGF, bFGF and Notch pathway components assay. Different samples were lysed in $0.2 \mathrm{ml}$ lysis buffer (0.1\% SDS, $1 \%$ NP-40, 50 mM HEPES, pH 7.4, 2 mM EDTA, $100 \mathrm{mM} \mathrm{NaCl}$, $5 \mathrm{mM}$ sodium orthovanadate, $40 \mu \mathrm{M}$ p-nitrophenyl phosphate, and $1 \%$ protease inhibitor mixture set I; Calbiochem). Lysates were centrifuged at $12,000 \mathrm{rpm}$ for $15 \mathrm{~min}$. The supernatant was collected and denatured. Protein concentrations were determined by the Bradford method (Bio-Rad Laboratories, Inc., Hercules, CA). Protein samples $(20 \mu \mathrm{g})$ were heated to $95^{\circ} \mathrm{C}$ for $5 \mathrm{~min}$, run on $10 \%$ SDS-PAGE gels, and transferred onto PVDF membranes (Millipore) using the semi-dry transfer method. The membranes were blocked for $1 \mathrm{~h}$ in Trisbuffered saline containing $0.01 \%$ Tween-20 with $10 \%$ non-fat dried milk, and incubated overnight at $4^{\circ} \mathrm{C}$ with the relevant antibodies (Santa Cruz Biotechnology, Inc.): anti-Notch-1, $-2,-3,-4$, Dll-1, -3, -4, Jag-1, -2, VEGF and bFGF antibodies. The membranes were rinsed and incubated for $1 \mathrm{~h}$ with the corresponding peroxidase-conjugated secondary antibodies. Chemiluminescent detection was performed using the ECL kit (Pierce). All bands from western blot analysis were analyzed using Image J software (version 1.6 NIH) to verify the relative level of Notch molecules, VEGF and bFGF markers compared to the internal control, $\beta$-actin. 
Measurement of MSC survival in ischemic myocardium. To measure MSC survival in the ischemic myocardium, the animals were euthanized on day 7 after transplantation. The survival of male donor MSCs that expressed the Sry gene in the female recipient hearts was calculated by using real-time PCR. Different numbers of male DNA (MSCs) were added to female DNA (mouse heart tissue) as standards: 100, 50, 25, 10, 5,1 and $0 \times 10^{4} \mathrm{MSCs} / 10 \mathrm{mg}$ heart tissue. The expression levels of Sry were quantified by normalizing the values relative to the mouse housekeeping gene, $\beta$-actin. The number of stem cells was calculated according to the calibration curve, which showed the threshold cycles $(\mathrm{Ct})$ of Sry expression against serially diluted MSCs (24).

Myocardial blood flow. Two weeks after transplantation and following the echocardiography study, measurements were taken to determine whether MSC ${ }^{\text {miR-126 }}$ increased blood flow to the infarcted area of mouse hearts. A total volume of $20 \mu \mathrm{l}$ $\left(7.2 \times 10^{4}\right)$ of $10 \mu \mathrm{m}$ blue fluorescent microspheres was injected into the left atrium. After $1 \mathrm{~min}$, a reference blood sample was withdrawn from the descending aorta at a rate of $1 \mathrm{ml} / \mathrm{min}$. The heart was then excised and weighed. The border, infarcted, and normal regions of the mouse hearts were dissected and analyzed separately. Microspheres were extracted from the blood and heart of each mouse by $0.2 \mathrm{ml}$ potassium hydroxide digestion, and fluorescence was measured with a 96-well plate reader. Myocardial blood flow (Qs) was calculated as Qs $(\mathrm{ml} / \mathrm{min} / \mathrm{g})=$ (As/Ar)Qr (ml/min)/Wt (g), where Qr represents the withdrawal rate of the reference blood, As and Ar represent the absorbance in sample tissue and reference blood, respectively, and $\mathrm{Wt}$ represents tissue weight (25). Blood flow in border and infarcted areas was expressed as a percentage of the normal myocardium.

Capillary density. To identify capillary density, the tissue sections $(5 \mu \mathrm{m})$ of the infarcted zone were stained with anti-VIII factor antibody (Santa Cruz Biotechnology, Inc.). Immunohistochemical staining was performed using the twostep immunohistochemical technique with DAB (Maixin-Bio, Fuzhou, China) as described in the manufacturer's specifications. After being restained with hematine, the samples were coverslipped and photographed. The capillaries were counted with a x200 microscopic objective in 10 randomly selected fields in 6 sections/animal (10 animals in each group) and averaged. Criteria for being counted consisted of lumen having diameters $<50 \mu \mathrm{m}$ and including single or tiny vascular with integral endothelial cells. Capillary density was expressed as the number of vessels per microscopic surface area $\left(0.95 \mathrm{~mm}^{2}\right)$.

Statistical analysis. Data re presented as the means \pm standard deviation. analysis of variance (ANOVA) with Scheffe's post hoc test was used to identify differences among all groups. A P-value of $<0.05$ was considered to indicate a statistically significant difference.

\section{Results}

Phenotypic characterization and differentiation capacity of cells. Cells were scattered in a number of colony distributions 3 days after planting. On days 8 and 9, the bottle was covered with long, spindle-like cells. The passaged cells (mostly spindle-
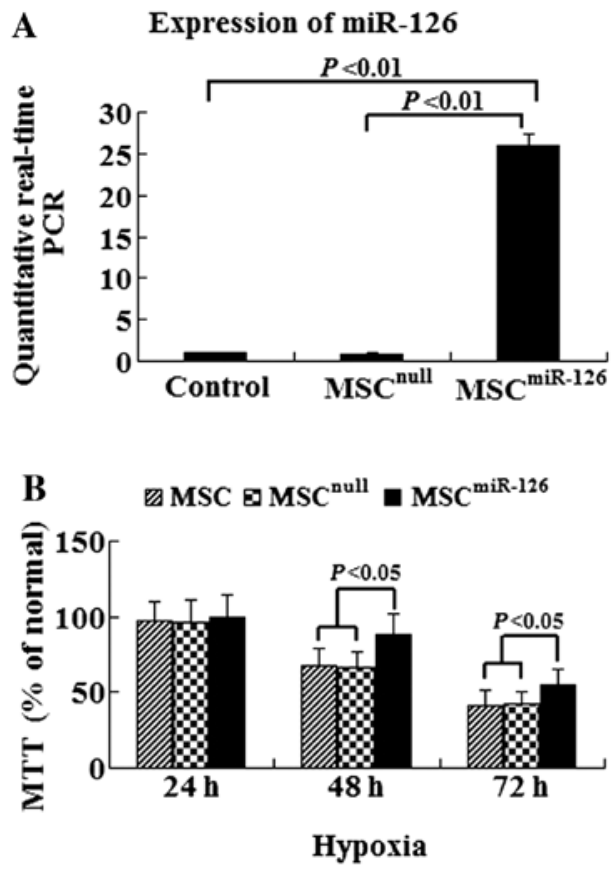

Figure 1. The efficiency of miR-126 transfection and the resistance of infected cells to hypoxia. (A) The efficiency of miR-126 transfection was analyzed by real-time PCR; miR-126 was evidently expressed in MSC ${ }^{\text {miR-126 }}$ group, but barely expressed in the control group and the $\mathrm{MSC}^{\text {null }}$ group. (B) (3,4,5-dimethylthiazol-2-yl)-2,5-diphenyltetrazolium bromide (MTT) intake by MSCs exposed to hypoxia for 24-72 h. (Control: MSCs, mesenchymal stem cells; MSC ${ }^{\text {null }}$, MSCs infected with mock vectors; MSC ${ }^{\text {miR-126, }}$ MSCs infected with miR-126 recombinant lentiviral vectors).

shaped or fibroblast-like cells) were uniformly distributed and covered the bottom every 4-5 days. They highly expressed the MSC surface marker molecules, CD29, CD90 and CD105, and negatively expressed the blood cell surface molecules, c-kit, CD34 and CD45. Following 3 weeks of adipogenic induction, the passaged cells stained positive for Oil Red O, showing a lipid-laden adipocyte phenotype. Similarly, when induced with osteogenic induction medium for 3 weeks, these cells showed osteogenesis upon staining with Alizarin Red S for calcium deposits. These results demonstrated that the stem cells posses pluripotent differentiation ability.

Efficiency of miR-126 transfection and resistance of infected cells to hypoxia. To determine the efficiency of gene transfection and the influence of miR-126 on MSC differentiation, a 10 -fold higher expression level is required. After infection with miR-126 recombinant lentiviral vectors, MSCs overexpressed eGFP. Quantitative real-time PCR data indicated that the efficiency of gene transfection in the MSC ${ }^{\text {miR-126 }}$ group was similar to that in the MSC $^{\text {null }}$ group (90.2 vs. 91.7\%). The expression of miR-126 in the MSC ${ }^{\text {miR-126 }}$ group was 26-fold higher than that in the MSC group $(\mathrm{P}<0.01)$, and 30-fold higher than the expression in the $\mathrm{MSC}^{\text {null }}$ group $(\mathrm{P}<0.01)$ (Fig. 1A). The TRE promoter induced by doxycycline was proved to be a strong cis-element in miRNA expression.

To examine the resistance of MSCs under hypoxic conditions, MSCs were exposed to hypoxia for 24-72 $\mathrm{h}$ and (3,4,5-dimethylthiazol-2-yl)-2,5-diphenyltetrazolium bromide (MTT) intake was assessed. In our experiment, MTT intake was not significantly different between the MSCs exposed to 

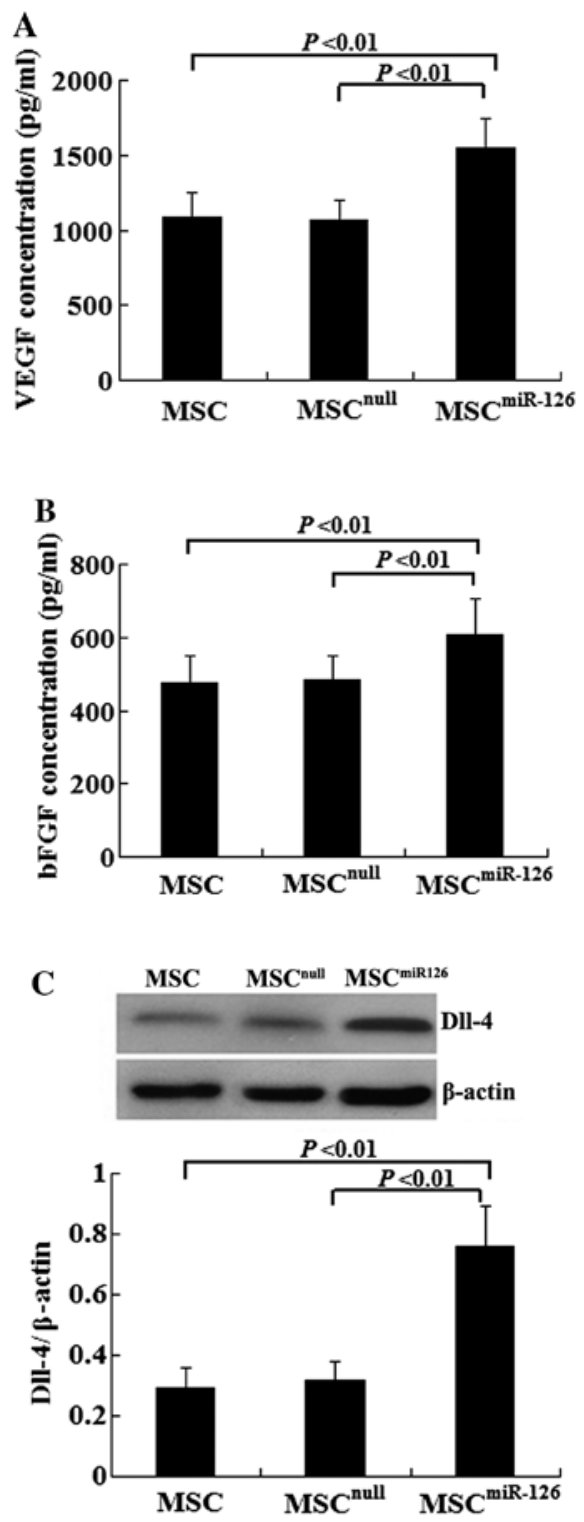

Figure 2. VEGF, bFGF and Notch ligand Dll-4 expression in vitro. (A and B) ELSIA analysis for (A) VEGF and (B) bFGF in MSC supernatants under hypoxic conditions; the levels of VEGF and bFGF in the $\mathrm{MSC}^{\mathrm{miR}-126}$ group were higher than those in the MSC and MSC ${ }^{\text {null }}$ groups. (C) Western blot analysis of Dll-4 in MSCs infected by lentiviral vectors encoding miR-126. Quantitative analysis shows that with the increased expression of miR-126, Dll-4 expression dramatically increased. (MSC, mesenchymal stem cells not infected with lentivirus; MSC $^{\text {null }}$, MSCs infected with mock vectors; MSC $^{\text {miR-126 }}$, MSCs infected with miR-126 recombinant lentiviral vectors).

hypoxia for $24 \mathrm{~h}$ and those in normal culture. However, it was significantly decreased when MSCs were exposed to hypoxia for 48 or $72 \mathrm{~h}$. Similar results were observed in the MSC ${ }^{\text {null }}$ group. Of note, the overexpression of miR-126 partially prevented this reduction (Fig. 1B).

Overexpression of miR-126 increases VEGF, bFGF and Dll-4 expression in vitro and in vivo. In vitro, there was no difference in the VEGF and bFGF concentration between the MSC ${ }^{\text {null }}$ group and the MSC group (VEGF, $1082.5 \pm 167.3$ vs. $1063.2 \pm 138.1$, $\mathrm{P}>0.05$; bFGF, $475.6 \pm 73.6$ vs. $482.4 \pm 67.3, \mathrm{P}>0.05$ ). However, MSCs overexpressing miR-126 had an increased secretion of

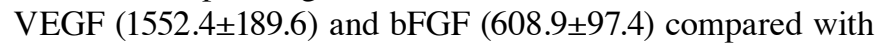

the $\mathrm{MSC}^{\text {null }}(\mathrm{P}<0.01)$ or the MSC group $(\mathrm{P}<0.01)$ under hypoxic conditions (Fig. 2A and B). We also examined Notch signaling, which plays an important role in angiogenesis. The expression of Notch-1,-2, -4, Dll-1, -4 and Jag-1 in the MSCs was examined by western blot analysis. There was no significant difference in the expression of Notch-1, -2, -4, Dll-1 or Jag-1 in the MSC ${ }^{\text {miR- }}$ ${ }^{126}$ group compared to the MSCs and $\mathrm{MSC}^{\text {null }}$ group. However, the Dll-4 protein level was dramatically increased with the increased expression of miR-126 in the MSCs (Fig. 2C).

Similarly, no difference was observed in the expression of VEGF and bFGF proteins between the MSC ${ }^{\text {null }}$ and the MSC group on days 7 and 14 after transplantation (all $\mathrm{P}>0.05)$ in vivo. However, the protein levels of VEGF and bFGF in the MSC or the MSC $^{\text {null }}$ group significantly increased on day 7 compared with the PBS group (all $\mathrm{P}<0.05$ ), and obvious differences were also detected on day 14 (all $\mathrm{P}<0.05$ ). MSCs modified with miR-126 demonstrated an upregulated expression of VEGF and bFGF proteins, which was significantly higher than that in the MSC $(\mathrm{P}<0.05)$, the $\mathrm{MSC}^{\text {null }}(\mathrm{P}<0.05)$, or the PBS group $(\mathrm{P}<0.01)$ (Fig. 3A-D). In addition, 7 days after transplantation, the expression level of Dll-4 protein in the infarcted tissue was also significantly higher in the MSCs ${ }^{\text {miR-126 }}$ group than in the PBS $(\mathrm{P}<0.001)$, the MSC $(\mathrm{P}<0.01)$, or the $\mathrm{MSC}^{\text {null }}$ group $(\mathrm{P}<0.01)$ (Fig. 3A, B and E). Similar effects were observed at 2 weeks post-transplantation. The level of Dll-4 in the MSC and the MSC $^{\text {null }}$ group increased compared to the PBS group at the 2 time-points (both $\mathrm{P}<0.05$ ); however, no statistically significant difference was observed between the MSC ${ }^{\text {null }}$ and the MSC group $(\mathrm{P}>0.05)$.

MSC survival in ischemic myocardium. To identify transplanted MSC survival in the ischemic myocardium, we established a calibration curve of the number of MSCs in recipient hearts vs. Sry gene expression (Fig. 4A). Ct values of the Sry gene were plotted on a semilogarithmic scale of MSC number to obtain a balanced contribution of all reference dilutions. Subsequently, hearts (4 in each group) were harvested 7 days after their respective treatment. In comparison with the MSC $^{\text {null }}$ or the MSC group, the expression of the Sry gene in the peri-infarcted and infarcted myocardium was significantly higher in the $\mathrm{MSC}^{\mathrm{miR}-126}$ group. However, no Sry expression was detected in the medium-control animals. The number of MSCs was calculated from the above calibration curve. The expression of miR-126 significantly increased MSC survival in the ischemic myocardium (Fig. 4B).

Transplantation of MCSs overexpressing miR-126 enhances angiogenesis and tubulogenesis. Semiquantitative analysis showed that 2 weeks after transplantation, the number of mature vessels in the peri-infarcted and infarcted tissue was significantly increased in the MSC $(25.3 \pm 3.6, \mathrm{P}<0.01)$, the $\operatorname{MSC}^{\text {null }}(24.2 \pm 4.5, \mathrm{P}<0.01)$ and the $\mathrm{MSC}^{\text {miR-126 }}$ group $(35.7 \pm 5.7$, $\mathrm{P}<0.001)$ compared to the PBS group $(11.2 \pm 1.6)$. No statistically significant difference was observed between the MSC $\mathrm{Cull}^{\text {nul }}$ and the MSC group $(\mathrm{P}>0.05)$. However, the mature microvessel number was significantly greater in the $\mathrm{MSC}^{\text {miR-126 }}$ than in the MSC $(\mathrm{P}<0.01)$ or $\mathrm{MSC}^{\text {null }}$ group $(\mathrm{P}<0.01)$ (Fig. 5).

In addition, to determine whether Notch ligand Dll-4 play a key role in tubulogenesis, we used shRNA to repress the expression of the Dll-4 gene in MSC ${ }^{\text {miR-126 }}$ cultures. Two 

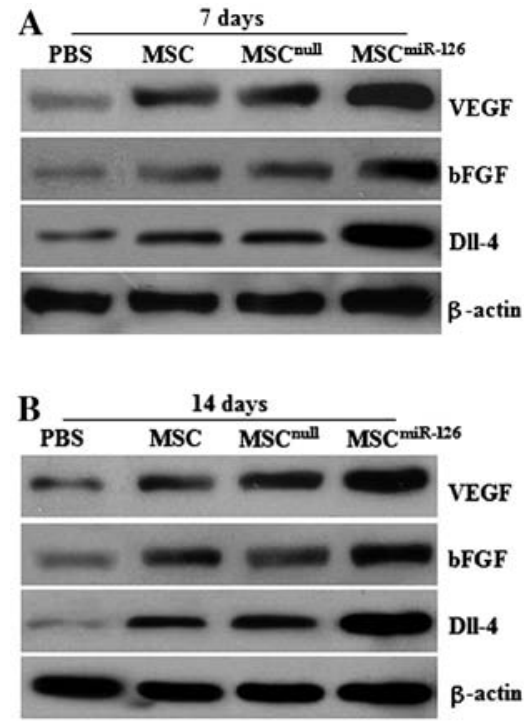

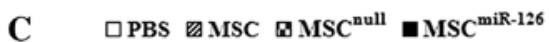
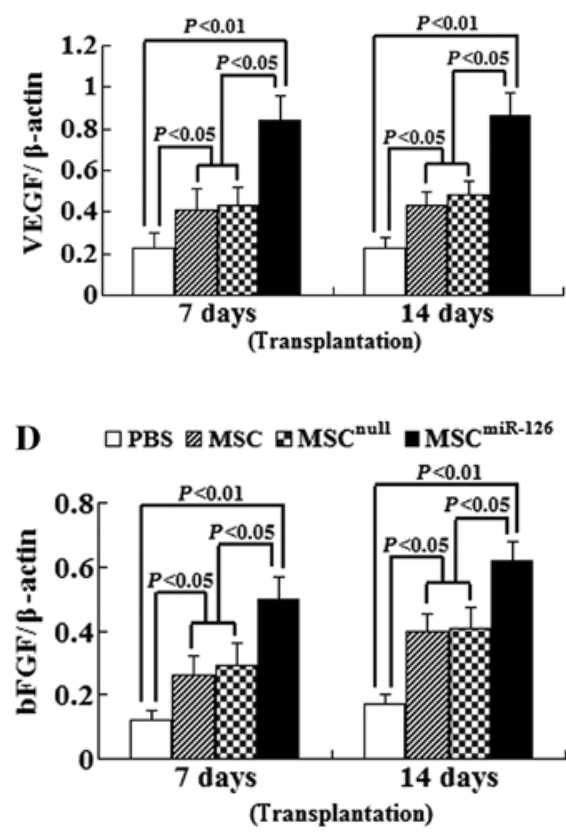

E $\quad \square$ PBS $\square$ MSC $\square$ MSC $^{\text {null }} \square$ MSC $^{\text {miR-126 }}$

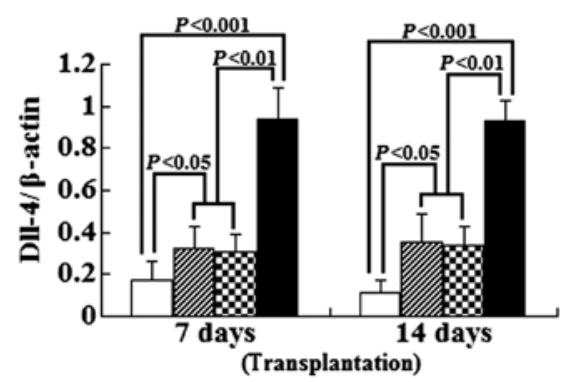

Figure 3. Expression levels of VEGF, bFGF and Dll-4 in vivo. (A and B) Western blot analysis was performed for VEGF, bFGF and Dll-4 expression in injured cardiac tissues at 7 and 14 days after transplantation. (C-E) Quantitative analysis shows that the expression levels of VEGF, bFGF and Dll-4 in the MSC and MSC ${ }^{\text {null }}$ group significantly increased compared with the PBS group. Moreover, MSCs modified with miR-126 showed an upregulation of the expression of the above molecules. (PBS, group injected with phosphate-buffered saline; MSC, MSCs not infected with lentivirus; MSC $^{\text {null }}$, MSCs infected with mock vectors; MSC $^{\text {miR-126 }}$, MSCs infected with miR-126 recombinant lentiviral vectors; 10 in each group).
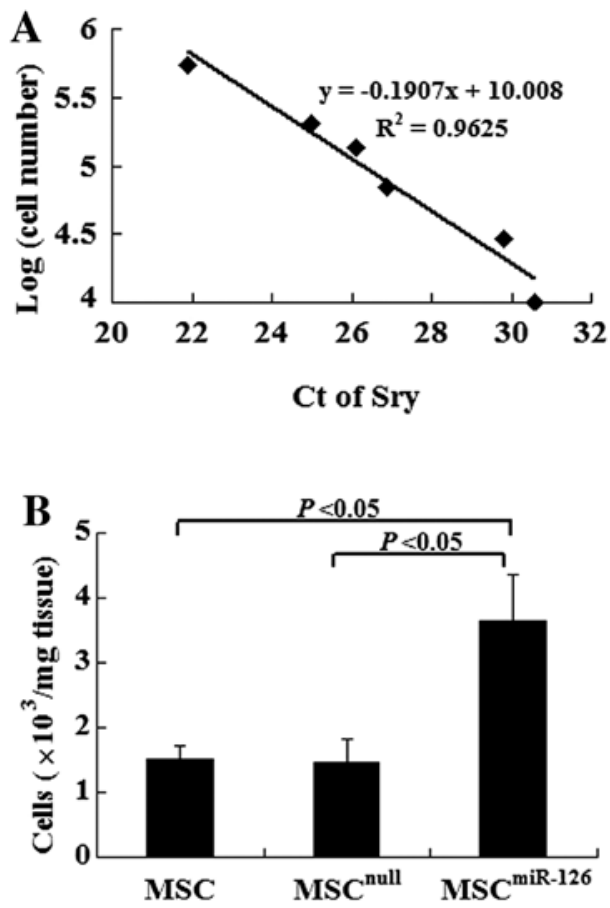

Figure 4. Engraftment of $\mathrm{MSC}^{\mathrm{miR}-126}$ in the ischemic myocardium 7 days after transplantation. (A) Calibration curve showing the ratio between threshold cycles $(\mathrm{Ct})$ of the Sry gene and MSC number. (B) Number of MSCs in the ischemi border and infarcted areas. (MSC, mesenchymal stem cell).

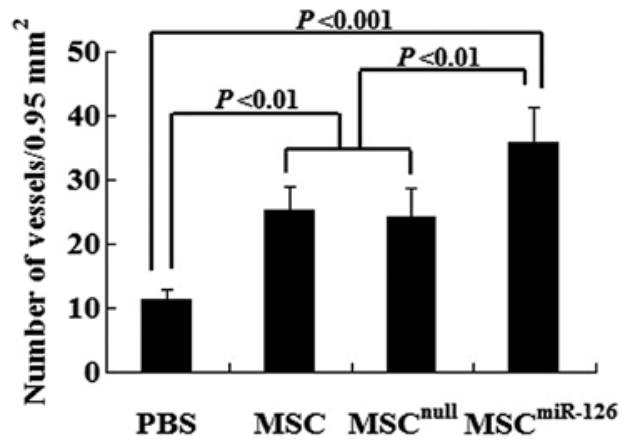

Figure 5. Immunohistochemical analysis of VIII factor-positive microvessels in risk areas 2 weeks after injection of MSCs or medium in the ischemic border zone. While less capillary growth was observed in the PBS group, the number of mature microvessels in the ischemic border and infarcted area of the mouse hearts was significantly increased in the cell-treated groups. However, the mature microvessel number was significantly greater in the $\mathrm{MSC}^{\text {miR-126 }}$ group than in the MSC and the $\mathrm{MSC}^{\text {null }}$ group. (PBS, group injected with phosphate-buffered saline; MSC, MSCs not infected with lentivirus; MSC $^{\text {null }}$, MSCs infected with mock vectors; MSC $^{\text {miR-126 }}$, MSCs infected with miR-126 recombinant lentiviral vectors; 10 in each group).

weeks after the transplantation of MSC ${ }^{\text {miR-126 }}$ cultures in which the expression of Dll-4 had been knocked down, we found increased immature vessel proliferation in the peri-infarcted area of mouse hearts. However, mature blood vessels with lumen were barely detected. This indicates that Dll-4 contributes to functional angiogenesis.

Transplantation of miR-126-transfected MCSs increases blood flow in ischemic myocardium. To determine whether new blood vessels translate to increased coronary blood flow to the 


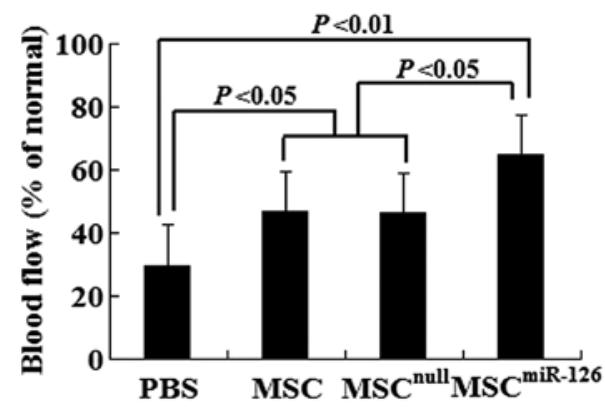

Figure 6. Analysis of blood flow in the ischemic area (including infarcted and border zone). Quantitative data suggested that $\mathrm{MSC}^{\text {miR-126 }}$ treatment significantly increased the blood flow to the ischemic tissue 2 weeks after transplantation. (PBS, group injected with phosphate-buffered saline; MSC, MSCs not infected with lentivirus; MSC ${ }^{\text {null }}$, MSCs infected with mock vectors; MSC $^{\text {miR-126 }}$, MSCs infected with miR-126 recombinant lentiviral vectors; 10 in each group).

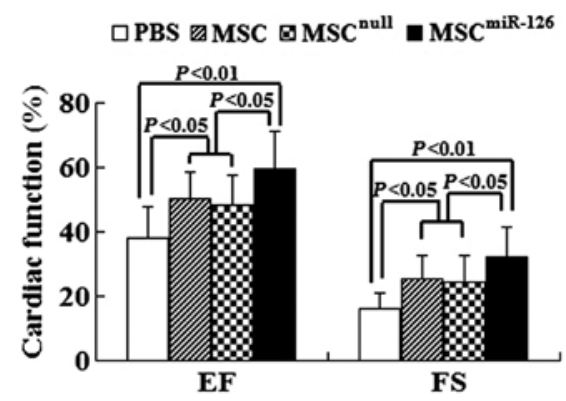

Figure 7. Assessment of cardiac function by echocardiography. Ejection fraction (EF) and fractional shortening (FS) were compared among the different treatments 14 days post-transplantation. (PBS, group injected with phosphate-buffered saline; MSC, MSCs not infected with lentivirus; MSC ${ }^{\text {null }}$ MSCs infected with mock vectors; MSC $^{\text {miR-126 }}$, MSCs infected with miR-126 recombinant lentiviral vectors; 10 in each group).

infarcted myocardium, we identified functional microvessels in the infarcted hearts using the the fluorescent microsphere method for regional blood flow assessment 2 weeks after transplantation. There was a significant decrease in blood flow to the infarcted and peri-infarcted zone. However, MSC and MSC ${ }^{\text {null }}$ treatment increased it to almost $47 \%$ of the normal levels. Of note, the transplantation of $\mathrm{MSC}^{\mathrm{miR}-126}$ further increased the blood flow to almost 65\% of the normal levels (Fig. 6).

Overexpression of miR-126 in MCSs for transplantation stimulates the improvement of cardiac function. Global heart function was assessed by echocardiography at 2 weeks post-transplantation. The results showed that $\mathrm{LV}$ function in infarcted C57BL/6 mice in the PBS group [EF (\%), 38.25 \pm 9.36 ; FS $(\%), 16.31 \pm 4.55]$ was significantly reduced compared to

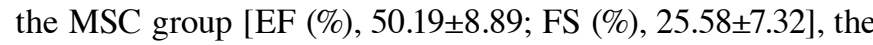
$\mathrm{MSC}^{\text {null }}$ group [EF (\%), 48.32 $\left.\pm 9.37 ; \mathrm{FS}(\%), 24.29 \pm 8.26\right]$ and the $\mathrm{MSC}^{\text {miR-126 }}$ group [EF (\%), 59.37 $\left.\pm 10.71 ; \mathrm{FS}(\%), 32.41 \pm 8.75\right]$. EF and FS in the MSC group and the MSC $^{\text {null }}$ group were evidently higher than those in the PBS group (all $\mathrm{P}<0.05$ ). In comparison with the PBS group, EF and FS in the $\mathrm{MSC}^{\text {miR-126 }}$ group were significantly improved (both $\mathrm{P}<0.01$ ). Furthermore, animals transplanted with $\mathrm{MSC}^{\mathrm{miR}-126}$ showed a marked improvement in
EF and FS compared with transplantation of MSC or MSC $\mathrm{Mull}^{\text {nul }}$ (all $\mathrm{P}<0.05$ ) (Fig. 7).

\section{Discussion}

Cell-based therapy appears to be a useful modality for myocardial repair and reverse remodeling in ischemic heart disease (26). The direct injection of isolated cardiomyocytes into injured tissue fails to enhance heart function since these cells do not thrive or integrate within the recipient heart (27). Bone marrow-derived MSCs have been proposed as a novel therapeutic approach for the improvement of infracted heart function through the regeneration of the myocardium or neovascularization $(28,29)$. However, low survival and angiogenic potential of transplanted cells influences the outcome of MSCs transplantation for the therapy of ischemic heart disease. Cell sheet grafts with genetically engineered properties to prolong cell survival and promote blood vessel networks integrated with pre-existing coronary may offer a potential approach to repair the dead or injured myocardium after MI.

We characterized the bone marrow-derived MSCs by immunophenotyping and confirmed that MSCs can be obtained from mouse bone marrow by serial passage of adherent cells. MSCs can be transfected with lentiviral vectors ecoding miR-126 with high efficiency and without any adverse effects on cell viability. MSCs modified with miR-126 can survive for long periods of time under hypoxic conditions and effectively express miR-126 for at least 2 weeks in the injected area.

Transplanted MSCs can be identified in the ischemic area by eGFP or Y-chromosome FITC staining after transplantation. However, it is not easy to compare the difference in survival rate of various MSCs. A number of previous studies have evaluated stem cell survival by observing Sry gene expression in the LV or the whole heart $(25,30,31)$. Since the Sry gene is located on the $\mathrm{Y}$ chromosome, the gene-positive stem cells can be inferred to be of male origin. However, Dresske et al (32) reported that the Sry gene-containing part was mainly localized in the LV adjacent to the infarcted area after MSCs were injected into the ischemic region. In our study, the expression of the Sry gene in the entire LV was extremely low. We assessed the survival of MSCs in the ischemic myocardium at 7 days post-transplantation, based on the expression of the Sry gene in the region. To ensure that all transplanted male MSCs were harvested, we collected enough ischemic tissue including the border and infarcted zone. To avoid uneven MSC distribution, MSCs were multiply injected into the peri-infarcted area.

Angiogenesis is the important process by which new vessels form through the growth of existing vessels, and includes the proliferation, sprouting, migration and tubulogenesis of endothelial cells, followed by pruning and remodeling of the vascular network. It is well known that VEGF and bFGF are major pro-angiogenic growth factors that play a pivotal role in angiogenesis. They can also activate several downstream pathways, including the mitogen-activated protein kinase (MAPK) and PI3K pathways, to regulate cell motility, proliferation and survival $(33,34)$. miRNAs, first known as regulators of development in worms and fruit flies, now act as important modulators of mammalian cardiovascular development and disease. miR-126 promotes angiogenesis in response to angiogenic growth factors, such as VEGF, bFGF and epidermal 
growth factor (EGF), by repressing negative regulators of signal transduction pathways. Thus, it plays an essential role in neoangiogenesis after MI and in the maintenance of vascular integrity (14). Of note, our data demonstrate that compared with the MSC and MSC ${ }^{\text {null }}$ cultures, MSC ${ }^{\text {miR-126 }}$ cultures significantly increased the secretion of angiogenic factors, such as VEGF and bFGF in vitro and in vivo. Paracrine effects include the humoral stimulation of the preservation of pre-existing cells and play a pivotal role in $\mathrm{MSC}^{\mathrm{miR}-126}$-mediated blood vessel formation. Both of the factors released from the modified transplanted stem cells promote angiogenesis in the ischemic area and represent another therapeutic strategy for cardiac repair. However, it remains unclear as to how the overexpression of miR-126 increases the paracrine effects of MSCs.

Our study shows that the intra-myocardial injection of MSCs overexpressing miR-126 into infracted area significantly enhances microvessel formation in ischemic tissue. Our results demonstrated a significantly greater capacity to form functional microvessels and increase blood flow to the infarcted and peri-infarcted zone in the group receiving MSCs modified with miR-126 than in the group transplanted with untreated MSCs. In tandem with the continuous improvement in capillary denisty, the transplantation of MSCs overexpressing miR-126 further enhanced the cardiac function in MI animals; this effect was superior to that exerted by the untransduced MSCs. At 2 weeks post-transplantation, the cardiac function in the MSC ${ }^{\text {miR-126 }}$ group gradually recovered, whereas the MSC group or the $\mathrm{MSC}^{\text {null }}$ group did not exhibit such evident effects. This suggests that the increase in adequately functional vessels and the enhancement of tissue perfusion play a key role in the improvement of cardiac function.

The VEGF pathway and Notch signaling are perhaps two of the most important mechanisms in the regulation of vascular development. As one of the Notch ligands, Dll-4 functions as an essential regulator in angiogenesis and tubulogenesis. The predominant site of Dll-4 expression is the vasculature, particularly the arteries, arterioles and capillaries during embryonic development, while in adults its expression is mostly restricted to small arteries and capillary networks $(16,35)$. Dll-4 plays a role in selecting tip cells in sprouting and appears to function as an anti-angiogenic branch factor, negatively regulating proangiogenic factors and positively regulating vessel maturation factors $(36,37)$. In our study, Dll-4 dramatically increased with the increased expression of miR-126 in vitro. The treatment of MSCs overexpressing miR-126 led to upregulation of Dll-4 in the ischemic myocardium. However, the transplantation of Dll-4-silenced MSC ${ }^{\text {miR-126 }}$ cultures to the infarcted heart induced the proliferation of immature vessels with no lumen and resulted in poor tissue perfusion, which was consistent with previous reports (38-40). Vascular network formation is coordinated by VEGF and Dll-4; Dll-4 may act downstream of VEGF as a 'brake' on VEGF-mediated angiogenic sprouting (41). Our findings indicated that the transplantation of MSCs overexpressing miR-126 led to the upregulation of Dll-4, which inhibited the angiogenic branch and enhanced tubulogenesis in the ischemic myocardium, forming functional microvessels.

In brief, there are 3 main findings in the current study: i) the genetic modification of MSCs to overexpress miR-126 led to their increased resistance under hypoxic conditions; ii) miR-126-transduced MSCs increased the release of angio- genic factors; iii) MSCs overexpressing miR-126 activated Dll-4, which contributed to the enhanced tubulogenesis. Targeting the expression of miR-126 in the transplanted MSCs may be a novel therapeutic strategy for angiogenesis and to restore cardiac function following MI.

In conclusion, our data demonstrate that the overexpression of miR-126 results in the upregulation of VEGF, bFGF and Notch ligand Dll-4 in the MSCs, as well as in the improved survival of engrafted MSCs and enhanced functional angiogenesis in the ischemic myocardium. MSCs genetically modified with miR-126 thus be represent a promising approach in neoangiogenesis and thereby the treatment of ischemic heart disease.

\section{Acknowledgements}

This study was supported by a grant from the National Nature Scientific Funding of China, 2009 (no. 30871053).

\section{References}

1. Feygin J, Mansoor A, Eckman P, Swingen C and Zhang J: Functional and bioenergetic modulations in the infarct border zone following autologous mesenchymal stem cell transplantation. Am J Physiol Heart Circ Physiol 293: H1772-H1780, 2007.

2. Tang YL, Zhao Q, Qin X, et al: Paracrine action enhances the effects of autologous mesenchymal stem cell transplantation on vascular regeneration in rat model of myocardial infarction. Ann Thorac Surg 80: 229-237, 2005.

3. Stamm C, Westphal B, Kleine HD, et al: Autologous bonemarrow stem-cell transplantation for myocardial regeneration. Lancet 361: 45-46, 2003.

4. Kinnaird T, Stabile E, Burnett MS, et al: Local delivery of marrow-derived stromal cells augments collateral perfusion through paracrine mechanisms. Circulation 109: 1543-1549, 2004.

5. Meyer GP, Wollert KC, Lotz J, et al: Intracoronary bone marrow cell transfer after myocardial infarction: eighteen months follow-up data from the randomized, controlled BOOST (Bone marrow transfer to enhance ST-elevation infarct regeneration) trial. Circulation 113: 1287-1294, 2006.

6. Uemura $\mathrm{R}, \mathrm{Xu} \mathrm{M}$, Ahmad $\mathrm{N}$ and Ashraf $\mathrm{M}$ : Bone marrow stem cells prevent left ventricular remodeling of ischemic heart through paracrine signaling. Circ Res 98: 1414-1421, 2006.

7. Tang J, Xie Q, Pan G, Wang J and Wang M: Mesenchymal stem cells participate in angiogenesis and improve heart function in rat model of myocardial ischemia with reperfusion. Eur $\mathbf{J}$ Cardiothorac Surg 30: 353-361, 2006.

8. Kinnaird T, Stabile E, Burnett MS, et al: Marrow-derived stromal cells express genes encoding a broad spectrum of arteriogenic cytokines and promote in vitro and in vivo arteriogenesis through paracrine mechanisms. Circ Res 94: 678-685, 2004.

9. Zhao Y, Samal E and Srivastava D: Serum response factor regulates a muscle-specific microRNA that targets Hand2 during cardiogenesis. Nature 436: 214-220, 2005.

10. Zhao Y and Srivastava D: A developmental view of microRNA function. Trends Biochem Sci 32: 189-197, 2007.

11. Kertesz M, Iovino N, Unnerstall U, Gaul U and Segal E: The role of site accessibility in microRNA target recognition. Nat Genet 39: 1278-1284, 2007

12. $\mathrm{Wu} \mathrm{L}$ and Belasco JG: Let me count the ways: mechanisms of gene regulation by miRNAs and siRNAs. Mol Cell 29: 1-7, 2008.

13. Wang S, Aurora AB, Johnson BA, et al: The endothelial-specific microRNA miR-126 governs vascular integrity and angiogenesis. Dev Cell 15: 261-271, 2008.

14. Fish JE, Santoro MM, Morton SU, et al: miR-126 regulates angiogenic signaling and vascular integrity. Dev Cell 15: 272-284, 2008.

15. Nicoli S, Standley C, Walker P, Hurlstone A, Fogarty KE and Lawson ND: MicroRNA-mediated integration of haemodynamics and Vegf signalling during angiogenesis. Nature 464: 1196-1200, 2010. 
16. Duarte A, Hirashima M, Benedito R, et al: Dosage-sensitive requirement for mouse D114 in artery development. Genes Dev 18: 2474-2478, 2004.

17. Krebs LT, Xue Y, Norton CR, et al: Notch signaling is essential for vascular morphogenesis in mice. Genes Dev 14: 1343-1352, 2000.

18. Roca $\mathrm{C}$ and Adams RH: Regulation of vascular morphogenesis by Notch signaling. Genes Dev 21: 2511-2524, 2007.

19. Phng LK and Gerhardt H: Angiogenesis: a team effort coordinated by notch. Dev Cell 16: 196-208, 2009.

20. Artavanis-Tsakonas S, Rand MD and Lake RJ: Notch signaling: cell fate control and signal integration in development. Science 284: 770-776, 1999.

21. Grajales L, Garcia J, Banach K and Geenen DL: Delayed enrichment of mesenchymal cells promotes cardiac lineage and calcium transient development. J Mol Cell Cardiol 48: 735-745, 2010.

22. Krampera M, Pasini A, Rigo A, et al: HB-EGF/HER-1 signaling in bone marrow mesenchymal stem cells: inducing cell expansion and reversibly preventing multilineage differentiation. Blood 106: 59-66, 2005.

23. Wang H, Cao F, De A, et al: Trafficking mesenchymal stem cell engraftment and differentiation in tumor-bearing mice by bioluminescence imaging. Stem Cells 27: 1548-1558, 2009.

24. Pons J, Huang Y, Takagawa J, et al: Combining angiogenic gene and stem cell therapies for myocardial infarction. J Gene Med 11: 743-753, 2009.

25. Li H, Zuo S, He Z, et al: Paracrine factors released by GATA-4 overexpressed mesenchymal stem cells increase angiogenesis and cell survival. Am J Physiol Heart Circ Physiol 299: H1772-H1781, 2010.

26. Macia $\mathrm{E}$ and Boyden PA: Stem cell therapy is proarrhythmic. Circulation 119: 1814-1823, 2009.

27. Yuasa S and Fukuda K: Cardiac regenerative medicine. Circ J 72 (Suppl A): A49-A55, 2008.

28. Orlic D, Kajstura J, Chimenti S, et al: Bone marrow cells regenerate infarcted myocardium. Nature 410: 701-705, 2001.

29. Kutryk MJ and Stewart DJ: Angiogenesis of the heart. Microsc Res Tech 60: 138-158, 2003.
30. Sheikh AY, Lin SA, Cao F, et al: Molecular imaging of bone marrow mononuclear cell homing and engraftment in ischemic myocardium. Stem Cells 25: 2677-2684, 2007.

31. Terrovitis J, Lautamaki R, Bonios M, et al: Noninvasive quantification and optimization of acute cell retention by in vivo positron emission tomography after intramyocardial cardiac-derived stem cell delivery. J Am Coll Cardiol 54: 1619-1626, 2009.

32. Dresske B, El Mokhtari NE, Ungefroren H, et al: Multipotent cells of monocytic origin improve damaged heart function. Am J Transplant 6: 947-958, 2006.

33. Graupera M, Guillermet-Guibert J, Foukas LC, et al: Angiogenesis selectively requires the p110alpha isoform of PI3K to control endothelial cell migration. Nature 453: 662-666, 2008.

34. Kerbel RS: Tumor angiogenesis. New Engl J Med 358: 2039-2049, 2008.

35. Benedito R and Duarte A: Expression of Dll4 during mouse embryogenesis suggests multiple developmental roles. Gene Expr Patterns 5: 750-755, 2005.

36. Trindade A, Kumar SR, Scehnet JS, et al: Overexpression of delta-like 4 induces arterialization and attenuates vessel formation in developing mouse embryos. Blood 112: 1720-1729, 2008.

37. Williams CK, Li JL, Murga M, Harris AL and Tosato G: Up-regulation of the Notch ligand Delta-like 4 inhibits VEGFinduced endothelial cell function. Blood 107: 931-939, 2006.

38. Scehnet JS, Jiang W, Kumar SR, et al: Inhibition of Dll4-mediated signaling induces proliferation of immature vessels and results in poor tissue perfusion. Blood 109: 4753-4760, 2007.

39. Noguera-Troise I, Daly C, Papadopoulos NJ, et al: Blockade of Dll4 inhibits tumour growth by promoting non-productive angiogenesis. Nature 444: 1032-1037, 2006.

40. Ridgway J, Zhang G, Wu Y, et al: Inhibition ofDll4 signalling inhibits tumour growth by deregulating angiogenesis. Nature 444 : 1083-1087, 2006.

41. Suchting S, Freitas C, le Noble F, et al: The Notch ligand Deltalike 4 negatively regulates endothelial tip cell formation and vessel branching. Proc Natl Acad Sci USA 104: 3225-3230, 2007. 